\title{
The effect of ketorolac on biofilm of Staphylococcus epidermidis isolated from post-cataract endophthalmitis
}

\author{
Silvia Rossetti • Leonardo D'Alessandro • \\ Fernando Pellegrino • María Alejandra Carrasco
}

Received: 9 January 2012 / Accepted: 1 March 2012 / Published online: 13 March 2012

(C) The Author(s) 2012. This article is published with open access at SpringerLink.com

\begin{abstract}
Purpose The purpose of this study is to investigate the effect of ketorolac on biofilm formation of Staphylococcus epidermidis isolated from patients with post-cataract endophthalmitis.

Methods Forty S. epidermidis strains isolated from postoperative endophthalmitis were used for this study. Biofilms were grown on microtitre plates for $24 \mathrm{~h}$, dyed, and stained with crystal violet. The mean optical density (OD) and the OD ratio (ODr $=\mathrm{OD}$ of the treated biofilm/OD of the untreated biofilm) were used for quantification. The biofilms were incubated with $13 \mathrm{mM}$ ketorolac and without ketorolac for controls.

Results The biofilm ODs of the S. epidermidis isolates untreated and treated with ketorolac were significantly different
\end{abstract}

\author{
S. Rossetti $\cdot$ L. D’Alessandro \\ Infectious Diseases Unit, Fundación Oftalmológica Argentina \\ Jorge Malbrán, \\ Parera 164, \\ C1014ABD Ciudad Autónoma de Buenos Aires, Argentina

\section{F. Pellegrino} \\ Department of Ophthalmology, Hospital de Clínicas "José de San \\ Martín", Universidad de Buenos Aires, \\ Av. Córdoba 2351, \\ C1120AAR Ciudad Autónoma de Buenos Aires, Argentina
}

\section{A. Carrasco}

Department of Ophthalmology, Hospital Universitario de

Mendoza,

Paso de los Andes 3051,

M5502BLI Mendoza, Argentina

M. A. Carrasco $(\square)$

Sarmiento 1326. Luzuriaga,

M5516BGH Mendoza, Argentina

e-mail: macarras73@gmail.com
$(0.335 \pm 0.06$ versus $0.158 \pm 0.03$, respectively; mean \pm SD; $P<0.001)$. Ketorolac reduced $S$. epidermidis biofilm formation by $47.6 \%$.

Conclusions Ketorolac, at a concentration of $13 \mathrm{mM}$, significantly reduces the formation of biofilm by strains of $S$. epidermidis that caused endophthalmitis.

Keywords Ketorolac · NSAIDs · Endophthalmitis · Biofilm $\cdot$ Staphylococcus epidermidis

\section{Introduction}

Endophthalmitis is a devastating sight-threatening postsurgical ocular complication. It remains the major concern for all cataract surgeons. Trends in favorable pre- and perioperative prophylaxis practices are difficult to analyze because of the infrequent occurrence of postoperative infection.

Staphylococcus epidermidis is recognized as the most common organism isolated in post-cataract endophthalmitis [1]. We previously found that $75 \%$ of our S. epidermidis post-cataract endophthalmitis strains had the genotypic markers of virulence $(i c a \mathrm{~A}, i c a \mathrm{D}$, and $m e c \mathrm{~A})$ and were biofilm producers [2].

Nonsteroidal anti-inflammatory drugs (NSAIDs) like sodium salicylate decrease the production of biofilm and bacterial adhesion to contact lenses [3, 4]. Considering that ketorolac is commonly used to prevent ocular inflammation, pain, and miosis in the perioperative period of cataract surgery, we designed an in vitro study to investigate the effect of ketorolac on the formation of biofilm by $S$. epidermidis. 


\section{Methods}

\section{Microorganisms}

The present study included $40 \mathrm{~S}$. epidermidis strains isolated from postoperative endophthalmitis between the years 2000 and 2008. All isolates were initially identified by classic microbiological and standard biochemical methods. Stock cultures were kept frozen at $-75{ }^{\circ} \mathrm{C}$ in a brain heart infusion broth containing $25 \%$ glycerol. Two S. epidermidis reference strains were used as controls: the well-known slime-producing strain ATCC 35984 (RP62A) and the non-slime-producing strain ATCC 12228.
Table 1 Effect of ketorolac on $S$. epidermidis biofilm formation by microplate assay

\begin{tabular}{|c|c|c|c|}
\hline Strain & $\mathrm{OD}_{570}$ control $($ mean $\pm \mathrm{SD})$ & $\mathrm{OD}_{570}($ ketorolac $)($ mean $\pm \mathrm{SD})$ & $\mathrm{OD}_{\mathrm{R}}$ \\
\hline 1 & $0.382 \pm 0.06$ & $0.183 \pm 0.03$ & 0.479 \\
\hline 2 & $0.238 \pm 0.01$ & $0.115 \pm 0.01$ & 0.483 \\
\hline 3 & $0.305 \pm 0.01$ & $0.145 \pm 0.06$ & 0.475 \\
\hline 4 & $0.297 \pm 0.12$ & $0.129 \pm 0.03$ & 0.434 \\
\hline 5 & $0.365 \pm 0.04$ & $0.167 \pm 0.07$ & 0.457 \\
\hline 6 & $0.284 \pm 0.02$ & $0.115 \pm 0.04$ & 0.405 \\
\hline 7 & $0.267 \pm 0.08$ & $0.129 \pm 0.01$ & 0.483 \\
\hline 8 & $0.329 \pm 0.05$ & $0.156 \pm 0.08$ & 0.474 \\
\hline 9 & $0.396 \pm 0.05$ & $0.168 \pm 0.05$ & 0.424 \\
\hline 10 & $0.412 \pm 0.06$ & $0.203 \pm 0.09$ & 0.493 \\
\hline 11 & $0.265 \pm 0.06$ & $0.115 \pm 0.04$ & 0.434 \\
\hline 12 & $0.423 \pm 0.09$ & $0.198 \pm 0.11$ & 0.469 \\
\hline 13 & $0.398 \pm 0.04$ & $0.176 \pm 0.06$ & 0.442 \\
\hline 14 & $0.256 \pm 0.02$ & $0.133 \pm 0.04$ & 0.519 \\
\hline 15 & $0.411 \pm 0.11$ & $0.185 \pm 0.02$ & 0.450 \\
\hline 16 & $0.357 \pm 0.07$ & $0.164 \pm 0.04$ & 0.459 \\
\hline 17 & $0.276 \pm 0.03$ & $0.108 \pm 0.05$ & 0.391 \\
\hline 18 & $0.351 \pm 0.08$ & $0.139 \pm 0.01$ & 0.444 \\
\hline 19 & $0.397 \pm 0.06$ & $0.195 \pm 0.01$ & 0.491 \\
\hline 20 & $0.408 \pm 0.03$ & $0.201 \pm 0.03$ & 0.493 \\
\hline 21 & $0.325 \pm 0.09$ & $0.174 \pm 0.04$ & 0.535 \\
\hline 22 & $0.306 \pm 0.05$ & $0.151 \pm 0.04$ & 0.493 \\
\hline 23 & $0.409 \pm 0.08$ & $0.197 \pm 0.08$ & 0.482 \\
\hline 24 & $0.435 \pm 0.12$ & $0.138 \pm 0.01$ & 0.317 \\
\hline 25 & $0.298 \pm 0.07$ & $0.156 \pm 0.05$ & 0.523 \\
\hline 26 & $0.254 \pm 0.04$ & $0.125 \pm 0.02$ & 0.492 \\
\hline 27 & $0.306 \pm 0.02$ & $0.136 \pm 0.04$ & 0.444 \\
\hline 28 & $0.335 \pm 0.09$ & $0.145 \pm 0.02$ & 0.433 \\
\hline 29 & $0.408 \pm 0.12$ & $0.198 \pm 0.13$ & 0.485 \\
\hline 30 & $0.312 \pm 0.08$ & $0.162 \pm 0.04$ & 0.519 \\
\hline 31 & $0.319 \pm 0.08$ & $0.159 \pm 0.09$ & 0.498 \\
\hline 32 & $0.397 \pm 0.09$ & $0.117 \pm 0.01$ & 0.295 \\
\hline 33 & $0.243 \pm 0.03$ & $0.116 \pm 0.03$ & 0.477 \\
\hline 34 & $0.268 \pm 0.12$ & $0.131 \pm 0.05$ & 0.489 \\
\hline 35 & $0.305 \pm 0.05$ & $0.189 \pm 0.06$ & 0.620 \\
\hline 36 & $0.315 \pm 0.02$ & $0.176 \pm 0.02$ & 0.559 \\
\hline 37 & $0.403 \pm 0.1$ & $0.201 \pm 0.05$ & 0.5 \\
\hline 38 & $0.409 \pm 0.04$ & $0.207 \pm 0.06$ & 0.506 \\
\hline 39 & $0.298 \pm 0.05$ & $0.175 \pm 0.06$ & 0.587 \\
\hline 40 & $0.257 \pm 0.04$ & $0.162 \pm 0.07$ & 0.630 \\
\hline All strains & $0.335 \pm 0.06$ & $0.158 \pm 0.03$ & $0.476 \pm 0.07$ \\
\hline
\end{tabular}


Biofilm model

Biofilms were studied using the static microtitre plate model established by Christensen et al. [5]. Ketorolac tris salt (Sigma) was prepared as 2-M stock solutions in PBS.

The $S$. epidermidis isolates were prepared as follows: an overnight culture grown in trypticase soy broth (TSB) at $37^{\circ} \mathrm{C}$ was diluted to $1: 100$ in TSB. A total of $200 \mu 1$ of these cell suspensions was transferred to a sterile 96well polystyrene U-bottom microtitre plate in TSB alone (control group) or in fresh TSB with $13 \mathrm{mM}$ ketorolac added (ketorolac group). The plates were incubated aerobically for $24 \mathrm{~h}$ at $37{ }^{\circ} \mathrm{C}$. Media and planktonic cells were removed. Adherent biofilm was fixed with $95 \%$ ethanol and was stained with $100 \mu \mathrm{l}$ of $1 \%(w / v)$ crystal violet for $5 \mathrm{~min}$. Then, unbound crystal violet was removed, and the wells were washed three times with $300 \mu \mathrm{l}$ of sterile distilled water. The water was then cleared and the microtitre plate was air-dried for $2 \mathrm{~h}$. The mean optical density (OD) was used for quantification using a routine microtitre plate reader at a wavelength of $570 \mathrm{~nm}$. All biofilm experiments were performed three times for each isolate to minimize the variability in OD measurements. To measure changes in the thickness of the biofilm, a ratio was calculated of the biofilm OD of the isolate incubated with ketorolac against the biofilm OD of the same isolate without ketorolac: $\left(\mathrm{OD}_{\mathrm{R}}=\mathrm{OD}\right.$ of the treated biofilm/OD of untreated biofilm). The significance of differences $(P<0.05)$ was assessed using the Mann-Whitney $U$ test.

\section{Results}

Biofilm ODs of the $S$. epidermidis isolates untreated and treated with ketorolac were significantly different $(0.335 \pm$ 0.06 versus $0.158 \pm 0.03$, respectively; mean $\pm \mathrm{SD} ; P<$ $0.001)$. Ketorolac reduced the $S$. epidermidis biofilm by $47.6 \%$ or $0.476 \pm 0.07$ (mean $\pm \mathrm{SD}$ ) expressed as OD ratio (Table 1). This effect was present in the 40 clinical isolates of $S$. epidermidis tested (Fig. 1).

\section{Discussion}

The incidence of post-cataract surgery endophthalmitis varies considerably in the medical literature. An incidence of 1.11 per 1,000 surgeries in the USA has been reported [6], 0.48 per 1,000 surgeries in Sweeden [7], 1.4 per 1,000 surgeries in Canada [8], and 0.49 per 1,000 surgeries in Brazil [9]. Visual outcome after endophthalmitis is generally poor; half the patients of the Endophthalmitis Vitrectomy Study achieved a visual acuity of 20/40 or better by 9 months after treatment [10].

Microorganisms from the ocular and periocular surface may enter the anterior chamber during phacoemulsification, with reported rates of anterior chamber contamination as high as $21 \%$ [11]. In the Endophthalmitis Vitrectomy Study, $70 \%$ of microbiological isolates contained coagulasenegative micrococci [12]. The isolates involved are usually normal flora of the surface of the eye and surrounding mucosa, such as $S$. epidermidis, which are generally not
Fig. 1 The graph shows that ketorolac reduced the biofilm of all strains tested expressed as OD ratio

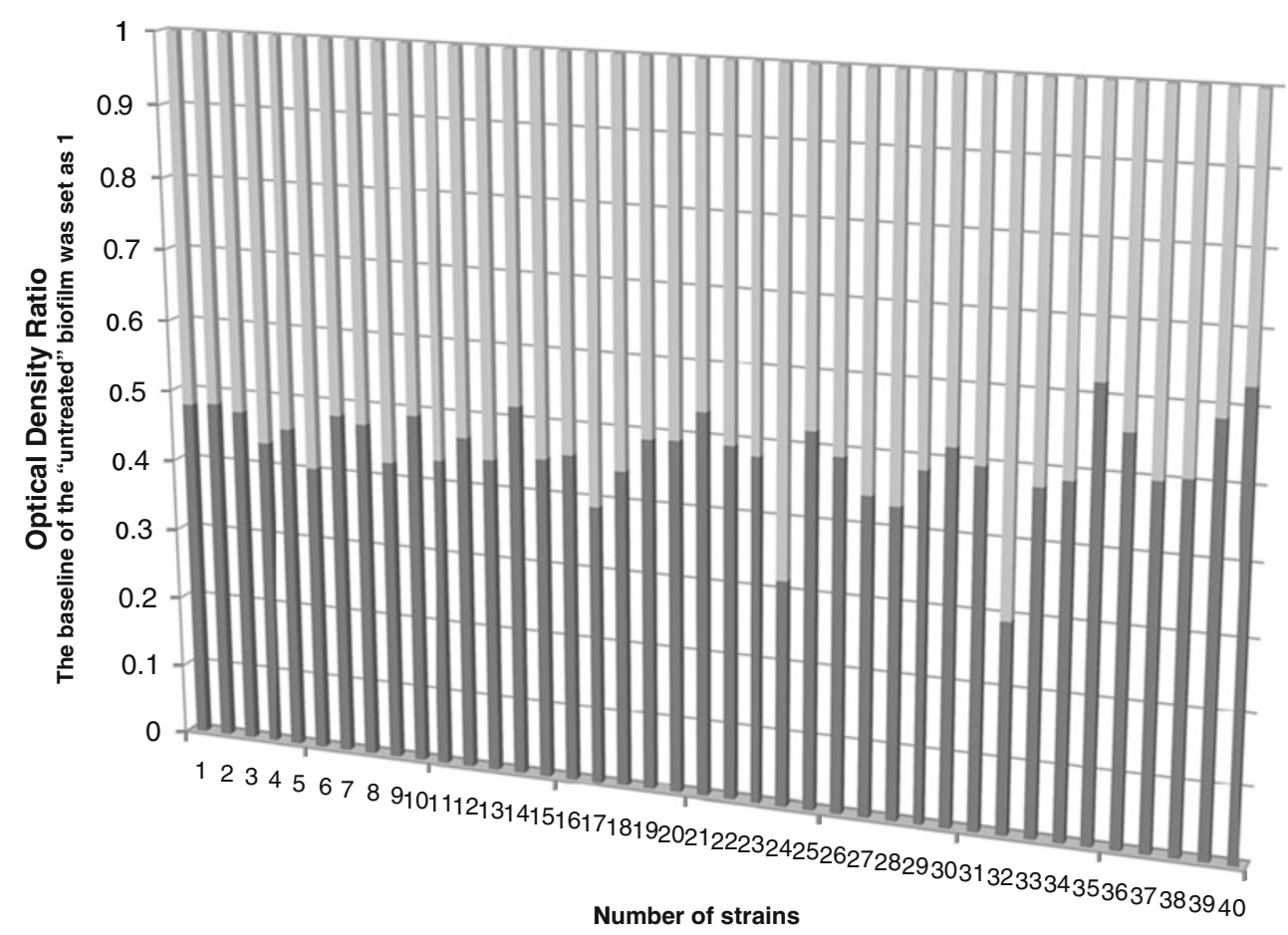


highly virulent. S. epidermidis has been isolated from $70 \%$ of normal eyes [13].

Biofilm is a microbially derived sessile community characterized by cells that are irreversibly attached to a substratum or interface or to each other, and are embedded in a matrix of extracellular polymeric substances that they have produced exhibiting an altered phenotype with respect to growth rate and gene transcription [14]. Formation of $S$. epidermidis biofilm is one of the most important virulence factors. Strains isolated from septic patients and prosthetic infections have the ica genes which mediate biofilm formation, and the mecA gene which is related to methicillin resistance. However, saprophytic isolates usually do not have these markers of virulence $[15,16]$.

This in vitro study shows that ketorolac reduces the formation of biofilm by $47.6 \%$ on all the strains tested. The mechanism by which ketorolac decreased biofilm production was not addressed in this study, and further research is needed for elucidation. The concentration of ketorolac used in our experiment is equivalent to approximately $0.5 \%$ ketorolac, the formulation present in commercially available products. Nonsteroidal anti-inflammatory drugs (NSAIDs) like salicylic acid and ketorolac can prevent adhesion and bacterial colonization of contact lenses [3]. Salicylic acid inhibited the production of teichoic acid, slime-associated proteins, and polysaccharide/adhesin production by S. epidermidis [17]. The adherence of bacteria to intraocular lenses (IOLs) during implantation and colonization of IOLs appears to have a role in the pathogenesis of postoperative endophthalmitis $[18,19]$. Herein is the importance for reducing biofilm production.

Most ophthalmic medications contain benzalkonium chloride as preservative. It has been shown that the presence of benzalkonium chloride at the minimal inhibitory is able to inhibit biofilm formation. However, it was able to induce biofilm development for the S. epidermidis at sub-MIC [20].

The failure of antibiotic treatment of biofilm-associated infections has led to a search for additive measures to eradicate bacteria within biofilms. Our findings support the hypothesis that the use of ketorolac in the perioperative period of cataract surgery may decrease the virulence of $S$. epidermidis, the most common microorganism involved in post-cataract endophthalmitis. Further studies are needed to prove this effect in vivo.

\section{Conclusion}

Ketorolac significantly reduces the formation of biofilm by strains of $S$. epidermidis that previously caused endophthalmitis. This effect may play a role in a new prophylactic strategy for postoperative endophthalmitis.
Financial disclosure None of the authors have any financial interest in any of the products described in this article.

Open Access This article is distributed under the terms of the Creative Commons Attribution License which permits any use, distribution and reproduction in any medium, provided the original author(s) and the source are credited.

\section{References}

1. Speaker MG, Milch FA, Shah MK et al (1991) Role of external bacterial flora in the pathogenesis of acute postoperative endophthalmitis. Ophthalmology 98:639-649

2. D'Alessandro L, Rossetti S (2002). Genotypic markers of Staphylococcus epidermidis virulence among strains isolated from postoperative endophthalmitis; in ARVO Meeting Abstracts December, p. 4433

3. Bandara BM, Sankaridurg PR, Willcox MD (2004) Non-steroidal anti inflammatory agents decrease bacterial colonisation of contact lenses and prevent adhesion to human corneal epithelial cells. Curr Eye Res 29:245-251

4. Farber BF, Hsieh HC, Donnenfeld ED et al (1995) A novel antibiofilm technology for contact lens solutions. Ophthalmology 102:831-836

5. Christensen GD, Simpson WA, Younger JJ et al (1985) Adherence of coagulase-negative staphylococci to plastic tissue culture plates: a quantitative model for the adherence of staphylococci to medical devices. J Clin Microbiol 22:996-1006

6. Keay L, Gower EW, Cassard SD, et al (2012) Postcataract surgery endophthalmitis in the United States analysis of the complete 2003 to 2004 medicare database of cataract surgeries. Ophthalmology (in press)

7. Lundstrom M, Wejde G, Stenevi U et al (2007) Endophthalmitis after cataract surgery: a nationwide prospective study evaluating incidence in relation to incision type and location. Ophthalmology $114: 866-870$

8. Hatch WV, Cernat G, Wong D et al (2009) Risk factors for acute endophthalmitis after cataract surgery: a population-based study. Ophthalmology 116:425-430

9. Melo GB, Bispo PJ, Regatieri CV et al (2010) Incidence of endophthalmitis after cataract surgery (2002-2008) at a Brazilian university-hospital. Arq Bras Oftalmol 73:505-507

10. Endophthalmitis Vitrectomy Study Group (1995) Results of the Endophthalmitis Vitrectomy Study. A randomized trial of immediate vitrectomy and of intravenous antibiotics for the treatment of postoperative bacterial endophthalmitis. Arch Ophthalmol 113:1479-1496

11. Srinivasan R, Gupta A, Kaliaperumal S et al (2008) Efficacy of intraoperative vancomycin in irrigating solutions on aqueous contamination during phacoemulsification. Indian J Ophthalmol 56:399-402

12. Han DP, Wisniewski SR, Wilson LA et al (1996) Spectrum and susceptibilities of microbiologic isolates in the Endophthalmitis Vitrectomy Study. Am J Ophthalmol 122:1-17

13. Perkins RE, Kundsin RB, Pratt MV et al (1975) Bacteriology of normal and infected conjunctiva. J Clin Microbiol 1:147-149

14. Donlan RM, Costerton JW (2002) Biofilms: survival mechanisms of clinically relevant microorganisms. Clin Microbiol Rev 15:167193

15. Galdbart JO, Allignet J, Tung HS et al (2000) Screening for Staphylococcus epidermidis markers discriminating between 
skin-flora strains and those responsible for infections of joint prostheses. J Infect Dis 182:351-355

16. Vandecasteele SJ, Peetermans WE, Merckx R et al (2003) Reliability of the ica, aap and atlE genes in the discrimination between invasive, colonizing and contaminant Staphylococcus epidermidis isolates in the diagnosis of catheter-related infections. Clin Microbiol Infect 9:114-119

17. Muller E, Al-Attar J, Wolff AG, Farber BF (1998) Mechanism of salicylate-mediated inhibition of biofilm in Staphylococcus epidermidis. J Infect Dis 177:501-503
18. Kodjikian L, Burillon C, Roques C et al (2003) Bacterial adherence of Staphylococcus epidermidis to intraocular lenses: a bioluminescence and scanning electron microscopy study. Invest Ophthalmol Vis Sci 44:4388-4394

19. Okajima Y, Kobayakawa S, Tsuji A, Tochikubo T (2006) Biofilm formation by Staphylococcus epidermidis on intraocular lens material. Invest Ophthalmol Vis Sci 47:2971-2975

20. Houari A, Di MP (2007) Effect of chlorhexidine and benzalkonium chloride on bacterial biofilm formation. Lett Appl Microbiol $45: 652-656$ 